(C) 2011 IEEE. Personal use of this material is permitted. Permission from IEEE must be obtained for all other uses, in any current or future media, including reprinting/republishing this material for advertising or promotional purposes, creating new collective works, for resale or redistribution to servers or lists, or reuse of any copyrighted component of this work in other works. 


\title{
Reduction of the Envelope Fluctuations of Multi-Carrier Modulations using Adaptive Neural Fuzzy Inference Systems
}

\author{
Víctor P. Gil Jiménez, Member, IEEE, Younes Jabrane, Ana García Armada, Senior Member, IEEE, \\ Brahim Ait Es Said, and Abdellah Ait Ouahman
}

\begin{abstract}
In this paper, a novel scheme for reducing the envelope fluctuations in multi-carrier signals applying Adaptive Neural Fuzzy Inference Systems (ANFIS) is proposed and analyzed. Once trained with signals with very low envelope fluctuations, such as those obtained by the Active Constellation Expansion - Approximate Gradient Project (ACE-AGP) algorithm, ANFIS approximately reaches a similar reduction as with ACE-AGP for multi-carrier signals without the complexity and the large convergence time of conventional ACE-AGP. We show that our approach is less complex than other previous schemes and with better performance.
\end{abstract}

Index Terms-OFDM, OFDMA, PAPR, cubic metric, ANFIS, ACE-AGP.

\section{INTRODUCTION}

$\mathbf{M}$ ULTI-CARRIER modulations such as Orthogonal Frequency Division Multiplexing (OFDM) and Orthogonal Frequency Division Multiple Access (OFDMA) have been advocated for Long Term Evolution (LTE) of wireless personal communications [1]. However, they exhibit large envelope f uctuations, which cause a loss in energy eff ciency due to the need of power back-off at the High Power Amplif ers (HPA). Since this problem is one of the most important drawbacks in multi-carrier modulations, there is a large number of proposals in the literature trying to reduce their envelope f uctuations [2].

Active Constellation Expansion (ACE) [3] is an interesting technique since it is able to achieve large reductions, it does not need side information and it only needs a small increase in transmit power. However, ACE requires many iterations for convergence and this, unfortunately, constitutes its main weakness. In this paper, ACE will be used to obtain the training set for the neural fuzzy system, with the aim of drastically reducing its implementation complexity.

To that end, we will use Adaptive Neural Fuzzy Inference System (ANFIS), a well-known tool to solve problems when

V. P. Gil Jiménez and A. García Armada are with the Department of Signal Theory and Communications, University Carlos III of Madrid, Spain (e-mail: \{vgil, agarcia\}@tsc.uc3m.es).

Y. Jabrane is with the Team of Telecommunications and Informatics Networks, University of Cadi Ayyad, Morocco (e-mail: y.jabrane@ucam.ac.ma).

B. A. Es Said is with the Team of Telecommunications and Informatics Networks, University of Cadi Ayyad, Morocco (e-mail: aitessaid@ucam.ac.ma).

A. A. Ouahman is with the Team of Telecommunications and Informatics Networks, University of Cadi Ayyad, Morocco (e-mail: ouahman@ucam.ac.ma).

This work has been partly funded by projects MULTI-ADAPTIVE (TEC2008-06327-C03-02), COMONSENS (CSD2008-00010) and AECI Program of Research Cooperation with Morocco (A/027714/09). the physical description of the underlying behavior is unknown, to provide some fuzzy (heuristics) rules that synthesize that behavior [4], [5].

The balance of this paper is organized as follows. Section II introduces the system model. In Section III, the neuro fuzzy systems proposed to reduce the envelope fuctuations are described and analyzed. Then, the obtained results are presented and discussed in Section IV. Finally, conclusions are drawn in Section V.

Note: Throughout the paper, the following notation will be used. Bold-face symbols will be used for vectors while normal-face for scalars. Time-domain signals will be denoted with small-case letters, whereas frequency-domain signals will use capitalized letters.

\section{SySTEM MODEL}

In a multi-carrier system, the time-domain complex baseband transmitted signal $\mathrm{x}^{\ell}$ for the $\ell$-th symbol can be written as

$\mathbf{x}^{\ell}=\left\{x^{\ell}[0] \cdots x^{\ell}[N-1]\right\}^{T}=\frac{1}{\sqrt{N}} \sum_{k=0}^{N-1} S_{k}^{\ell} e^{\frac{j 2 \pi k n}{N}}, \quad \ell=0 \cdots \infty$

where $N$ is the number of sub-carriers and $S_{k}^{\ell}$ is the frequencydomain complex base-band symbol modulated on the $k$-th subcarrier at OFDM symbol $\ell$.

\section{A. Cubic Metric}

The classical metric to evaluate the envelope fuctuations in OFDM is the Peak-to-Average Power Ratio (PAPR). However, this metric only accounts for the maximum peak and therefore, it may not take appropriately into account the distortion effect due to the non linear response of the High Power Amplif er. For this reason, other metrics have been recently proposed such as the Normalized Distortion (ND) [6] or Cubic Metric (CM) [7]. The CM uses higher-order statistics suitable to evaluate the power de-rating factor in a HPA, and it is def ned by the Third Generation Partnership Project (3GPP) as [7]

$$
C M=\frac{R C M(d B)-R C M_{r e f}(d B)}{K} d B
$$

where $R C M$ is the Raw Cubic Metric that for a signal $\mathbf{x}$ is def ned as

$$
R C M=20 \log _{10}\left(\sqrt{E\left\{\left(\frac{|\mathbf{x}|}{\sqrt{E\{(\mathbf{x})\}}}\right)^{3}\right\}}\right) d B
$$

$R C M_{r e f}$ is $1.52 \mathrm{~dB}$, and $K$ is 1.56 for multi-carrier systems. In this paper, the CM is used since it provides a better insight on envelope fuctuations. 


\section{B. ACE method}

The ACE method modif es and expands the constellation points within an allowable region which does not affect the demodulation slicer, and thus, it does not need side information to be sent. By using this new degree of freedom, multicarrier signals with arbitrarily low envelope fuctuations can be obtained. In [3], different algorithms to achieve PAPR reduction are provided. In this paper, the Approximate GradientProject (AGP) method will be used. The complexity of the algorithm, in terms of complex multiplications and additions per OFDM symbol, is $N_{\text {iter }} \times\left(2 N+N / 2 \log _{2}(N)\right)$ and $N_{\text {iter }} \times\left(4 N+N \log _{2}(N)\right)$, respectively, where $N_{\text {iter }}$ is the number of iterations, which is usually high. Besides, on each iteration, the PAPR or CM need to be evaluated to determine if the target goal has been reached. These operations are also required in many other methods [2]. Besides, a DFT/IDFT are needed per iteration.

\section{NEURO-FuZZY Systems}

A Fuzzy if then rule is an expression of the form IF A THEN $B$, where A and B are labels of fuzzy sets [4] characterized by appropriate membership functions. The proposed form of fuzzy if then rule by Takagi and Sugeno [4] has fuzzy sets involved only in the premise part. In this paper we use the typical fuzzy rule in a Sugeno fuzzy model [8], which has the format:

$$
\text { If } x \text { is } \mathcal{A} \text { and } y \text { is } \mathcal{B} \text { then } z=f(x, y)
$$

where $\mathcal{A}$ and $\mathcal{B}$ are fuzzy sets in the antecedent and $z=$ $f(x, y)$ is a crisp function in the consequent. We consider the f rst-order Sugeno fuzzy inference system which contains two rules:

$$
\begin{aligned}
& \text { If } x \text { is } \mathcal{A}_{1} \text { and } y \text { is } \mathcal{B}_{1} \text { then } f_{1}=p_{1} x+q_{1} y+r_{1} \\
& \text { If } x \text { is } \mathcal{A}_{2} \text { and } y \text { is } \mathcal{B}_{2} \text { then } f_{2}=p_{2} x+q_{2} y+r_{2}
\end{aligned}
$$

The fring strengths $\omega_{1}$ and $\omega_{2}$ are usually obtained as the product of the membership grades in the premise part, and the output $f$ is the weighted average of each rule's output.

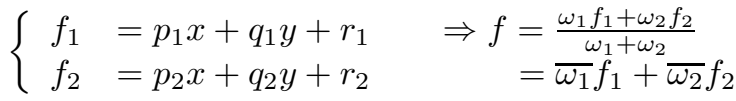

To facilitate the learning of the Sugeno fuzzy model, it is convenient to introduce the fuzzy model into the framework of adaptive networks that can systematically compute gradient vectors. The resultant network architecture is called ANFIS. Using a given input/output data set, ANFIS constructs a Fuzzy Inference System (FIS), whose membership function parameters are here tuned using a backpropagation algorithm in combination with Least Squares (LS) method. This technique provides a method for the fuzzy modeling procedure to learn information about a data set, in order to compute the membership function parameters that best allow the associated fuzzy inference system to track the given input/output data. The overall output $f$ can be expressed as linear combinations of the consequent parameters. In the following subsections, a Time-domain ANFIS system is developed to learn which time-domain signals exhibit low envelope fuctuations, and a Time-Frequency-domain ANFIS is proposed to learn which constellation regions are allowed or forbidden.
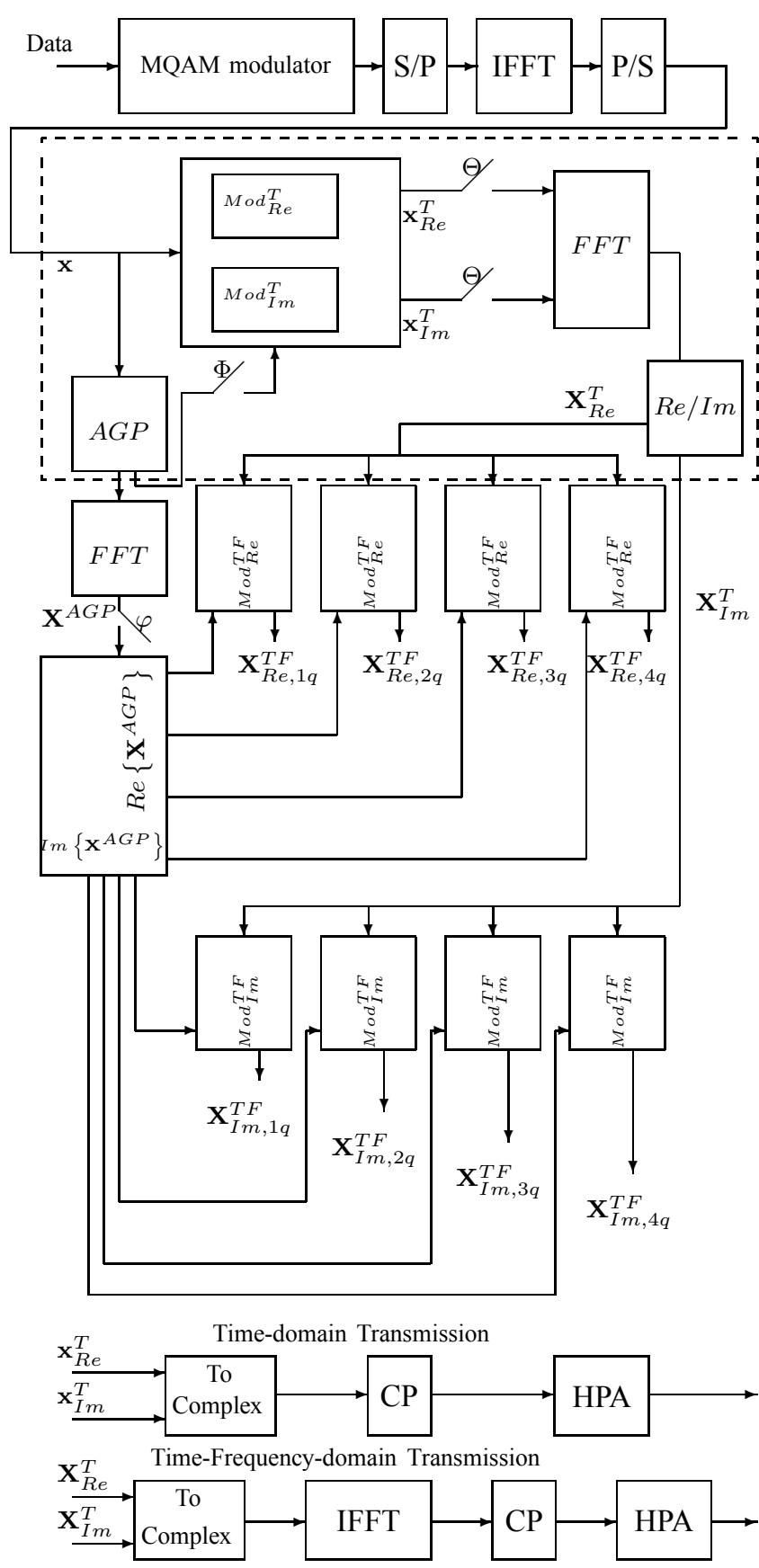

Fig. 1. Integrated transmission and training scheme for Timedomain Models $\left(\operatorname{Mod}^{T}\right)$ and Time-Frequency-domain Models $\left(\operatorname{Mod}_{1}^{T F}, \operatorname{Mod}_{2}^{T F}, \operatorname{Mod}_{3}^{T F}, \operatorname{Mod}_{4}^{T F}\right)$ for real and imaginary parts. Time-domain training: Switch $\Phi$ on, $\Theta$ off and $\varphi$ off. Time-Frequencydomain training: Switch $\Phi$ off, $\Theta$ on and $\varphi$ on. Running: $\Phi$ off, $\Theta$ on and $\varphi$ off.

\section{A. Time-domain ANFIS (T)}

The frst proposal is based on the time-domain OFDM signal. We train our ANFIS by using the signals with low envelope fuctuations obtained by the ACE-AGP algorithm. Since ANFIS only works with integer signals, we need to f rst decompose into real and imaginary parts the time-domain original signal. The training process is detailed in the following:

1) Use the original time-domain data $x$ as an input to the 


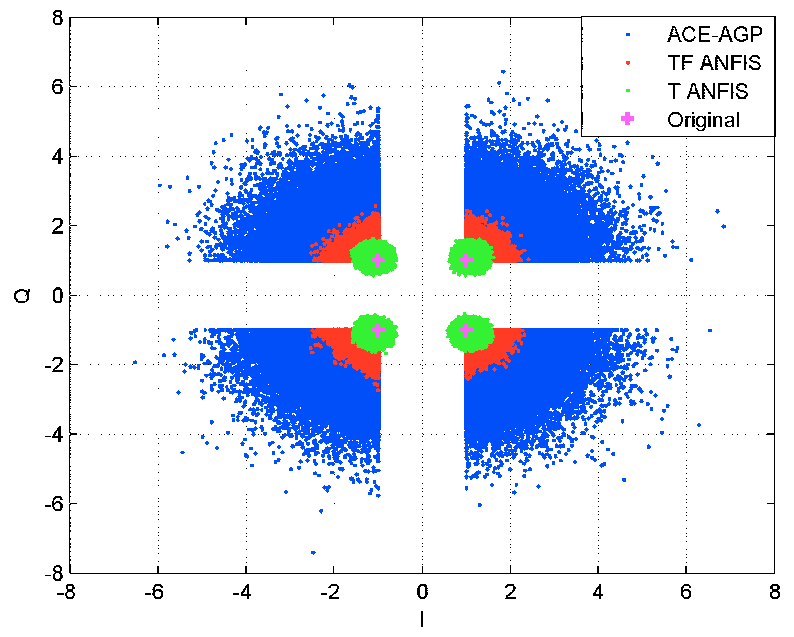

Fig. 2. Constellation after ACE-AGP and proposed Time-domain ANFIS and Time-Frequency-domain ANFIS. QPSK

ACE-AGP algorithm to obtain $\mathrm{x}^{A G P}$, i.e., a signal with reduced envelope fuctuations.

2) Split $\mathbf{x}$ and $\mathrm{x}^{A G P}$ into two sets, namely, the training set $\mathbf{x}^{t r}, \mathbf{x}^{A G P, t r}$ and the test set, $\mathbf{x}^{t s}, \mathbf{x}^{A G P, t s}$.

3) Decompose into real and imaginary parts the original data $\mathbf{x}^{t r}\left(\mathbf{x}_{R e}^{t r}, \mathbf{x}_{I m}^{t r}\right)$, and ACE-AGP output $\mathbf{x}^{A G P, t r}$ $\left(\mathbf{x}_{R e}^{A G P, t r}, \mathbf{x}_{I m}^{A G P, t r}\right)$.

4) Obtain $\mathbf{x}_{R e}^{T}$ and $\mathbf{x}_{I m}^{T}$ by training the two models $M o d_{R e}^{T}$ and $\operatorname{Mod}_{I m}^{T}$ with the pairs $\left[\mathbf{x}_{R e}^{t r}, \mathbf{x}_{R e}^{A G P, t r}\right]$ and $\left[\mathbf{x}_{I m}^{t r}\right.$, $\left.\mathbf{x}_{I m}^{A G P, t r}\right]$, respectively, in eq. (9), as $x=x_{\gamma}^{t r}[n]$ and $f=x_{\gamma}^{A G P, t r}$ for $n=0 \cdots N-1$ and $\gamma=R e$ or $\gamma=I m$.

5) Test with the values of $\mathbf{x}^{t s}$ to validate the models $\operatorname{Mod}_{R e}^{T}$ and $\operatorname{Mod}_{I m}^{T}$.

After training and testing the two neural fuzzy models (for the real and the imaginary parts, respectively), they are ready to be used. In Fig. 1, the proposed training scheme for time-domain neural fuzzy is shown inside the dashed box (also the whole transmission chain is depicted). After this off-line training (only needed when the number of sub-carriers or modulation type are changed), in operating mode, the AGP part in Fig. 1 is not needed. For clarity purposes, the implementation of ANFIS is detailed in the following equations, where $\eta$ denotes Real or Imaginary part for $\operatorname{Model}_{\eta}^{T}$, depending on the model.

At layer 1: Each node in this layer generates membership grades of linguistic labels $\left(\Omega_{i}^{j}\right.$ denotes the output of the $i$ th node in $j$ th layer)

$$
\Omega_{i}^{1}=\mu_{A_{i}}\left(x_{\eta}\right)=\exp \left(-\frac{\left(x_{\eta}-c_{i}\right)^{2}}{2 \sigma_{i}^{2}}\right)
$$

where $A_{i}$ is the linguistic label (small, large, etc.) associated with this node, $c_{i}$ and $\sigma_{i}^{2}$ are, respectively, the center and variance corresponding to the Gaussian membership function. At Layer 2: Each node in this layer calculates the fring strength of a rule via multiplication

$$
\Omega_{i}^{2}=\omega_{i}=\mu_{A_{i}}\left(x_{\eta}\right) * \mu_{B_{i}}\left(x_{\eta}\right)
$$

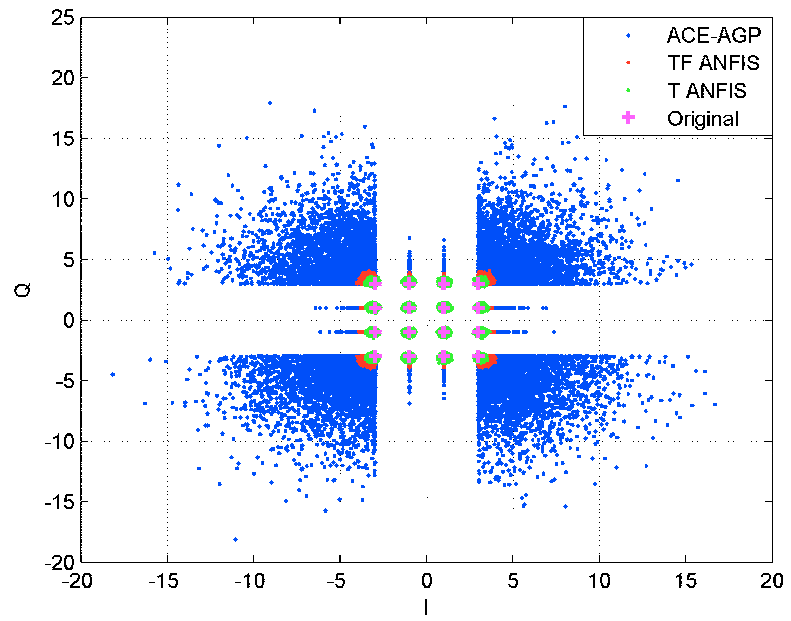

Fig. 3. Constellation after ACE-AGP and proposed Time-domain ANFIS and Time-Frequency-domain ANFIS. 16-QAM

At Layer 3: Node $i$ in this layer calculates the ratio of the ith rule's f ring strength

$$
\Omega_{i}^{3}=\frac{\omega_{i}}{\omega_{1}+\omega_{2}}=\bar{\omega}_{i}
$$

At Layer 4: Node $i$ in this layer computes the contribution of ith rule to the overall output

$$
\Omega_{i}^{4}=\bar{\omega}_{i}\left(p_{i} x_{\eta}+r_{i}\right)
$$

and f nally, the single node at Layer 5 computes the overall output

$$
\Omega^{5}=\left(\overline{\omega_{1}} x_{\eta}\right) p_{1}+\left(\overline{\omega_{2}} x_{\eta}\right) p_{2}+\bar{\omega}_{1} r_{1}+\bar{\omega}_{2} r_{2}
$$

The linear parameters $p_{i}$ and $r_{i}$, with $i=1,2, \ldots, n(n=2$ and $n=8$ for Time-domain and Time-Frequency-domain (later) schemes we are proposing, respectively), and the parameters $c_{j}, \sigma_{j}$ of the membership functions $(j=1,2, \ldots, s$, where $s$ is the number of fuzzy rules) of the neuro-fuzzy structure are adjusted by using the following equations

$$
\begin{aligned}
& p_{i}(t+1)=p_{i}(t)-\lambda \frac{\partial E}{\partial p_{i}} \\
& r_{i}(t+1)=r_{i}(t)-\lambda \frac{\partial E}{\partial r_{i}} \\
& c_{j}(t+1)=c_{j}(t)-\lambda \frac{\partial E}{\partial c_{j}} \\
& \sigma_{j}(t+1)=\sigma_{j}(t)-\lambda \frac{\partial E}{\partial \sigma_{j}}
\end{aligned}
$$

where $\lambda$ is the learning rate. At the end of this section, these parameters are derived.

In Fig. 2 and Fig. 3, the constellations obtained by ACEAGP and the proposed Time-domain ANFIS are presented for QPSK and 16-QAM modulations. It can be seen that, although they produce a similar performance (in terms of reduction of the envelope fuctuations), the constellations are different. In fact, some constellation points for Time-domain ANFIS in Fig. 2 and Fig. 3 are lying into not allowable regions, which will worsen the system Bit Error Rate (BER) performance, as it can be observed in Fig. 4. In this fgure, all the schemes (original, ACE-AGP and the two proposed ANFIS) have been evaluated in the presence of Additive White Gaussian Noise (AWGN) and a Travelling Wave Tube Amplifer (TWTA). 


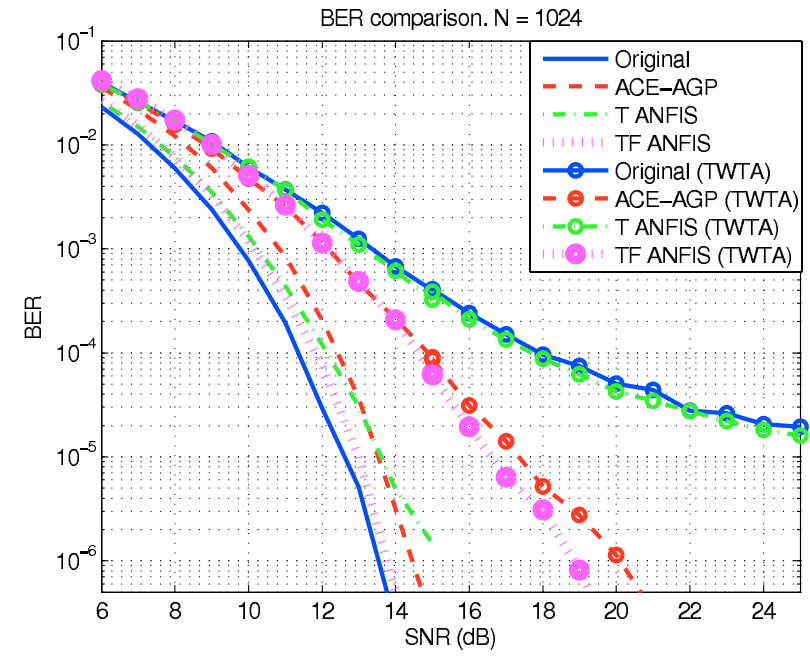

Fig. 4. BER performance for ACE-AGP and proposed Time-domain ANFIS and Time-Frequency-domain ANFIS. QPSK modulation. AWGN and TWTA.

It can be seen that the proposed Time-domain neural fuzzy outperforms ACE-AGP for low Signal to Noise Ratio (SNR) but not for high SNR. And moreover, both methods (ACEAGP and proposed Time-domain ANFIS) obtain a worse BER performance than the original non-processed signal. The reasons for this behavior are the following. Since all the systems have been simulated at the same SNR, the noise power will be def ned by the transmitted power. If transmitted power increases, so the noise power. Thus, for non-extended points in the constellation ( $70 \%$ of total in ACE-AGP), the noise power will be larger than in the original scenario and so, the BER increases. The average constellation energy for the original signal is 1 for QPSK (and 5 for 16-QAM), whereas for ACE-AGP it is 1.29 (5.73), and for the proposed Time-domain ANFIS it is $1.2(5.45)$. In the low SNR regime, since the proposed Time-Domain ANFIS concentrates more the energy than ACE-AGP, the performance is better in terms of BER. However, in the high SNR regime, the symbols placed into the not-allowable region ( $20 \%$ of total) degrade its performance.

\section{B. Time-Frequency-domain ANFIS (TF)}

The main problem with the Time-Domain ANFIS scheme is that the neural fuzzy is not able to learn which regions in the constellation are allowed and which ones are forbidden from the time-domain signal. Thus, a second ANFIS working on the frequency-domain is proposed. In this scheme, the already obtained signals with the frst Time-domain ANFIS are introduced to the second ANFIS (after transformation to the frequency-domain by using a DFT). The second ANFIS (working on the frequency-domain) is trained with the obtained frequency-domain ACE-AGP signals in order to learn which points in the constellation are allowed. Again, due to the limitation to integer signals, we work separately with the real and imaginary parts. The training procedure is as follows:

1) Apply DFT on $\mathbf{x}_{R e}^{T}$ and $\mathbf{x}_{I m}^{T}$ to obtain the frequencydomain signal $\mathbf{X}^{T}$.

2) Split in training samples $\mathbf{X}^{T, t r}$ and test samples $\mathbf{X}^{T, t s}$.

3) Separate the training samples $\mathbf{X}^{T, t r}$ in the four constellation regions in order to train eight ANFISs. We will divide the signal in two sets: 1 st set concerning real parts and the 2nd set, the imaginary parts, as it is shown in Fig. 1.

4) Train the frst set of ANFIS by $\Re e\left(\mathbf{X}^{T, t r}\right)$ to generate $\operatorname{Mod}_{R e, 1 q}^{T F}, \operatorname{Mod}_{R e, 2 q}^{T F}, \quad \operatorname{Mod}_{R e, 3 q}^{T F}$ and $\operatorname{Mod}_{R e, 4 q}^{T F}$ for each quadrant.

5) Train the second set of ANFIS by $\Im m\left(\mathbf{X}^{T, t r}\right)$ to generate $\operatorname{Mod}_{I m, 1 q}^{T F}, \operatorname{Mod}_{I m, 2 q}^{T F}, \operatorname{Mod}_{I m, 3 q}^{T F}$ and $\operatorname{Mod}_{I m, 4 q}^{T F}$ for each quadrant.

6) Test with the values of $\mathbf{X}^{T, t s}$ to validate the models.

The operations carried out at each layer are summarized in the following equations, where $\eta$ stands for Real or Imaginary part and $\varrho$ is the number of quadrant $(\varrho=1 \cdots 4)$ for Model ${ }_{\eta, \varrho q}^{T F}$.

\section{At Layer 1}

$$
\Omega_{i}^{1}=\mu_{A_{i}}\left(x_{\eta, \varrho q}^{T}\right)=\exp \left(-\frac{\left(x_{\eta, \varrho q}^{T}-c_{i}\right)^{2}}{2 \sigma_{i}^{2}}\right)
$$

at Layer 2

$$
\Omega_{i}^{2}=\omega_{i}=\mu_{A_{i}}\left(x_{\eta, \varrho q}^{T}\right) * \mu_{B_{i}}\left(x_{\eta, \varrho q}\right)
$$

at Layer 3

$$
\Omega_{i}^{3}=\frac{\omega_{i}}{\omega_{1}+\omega_{2}}=\bar{\omega}_{i}
$$

at Layer 4

$$
\Omega_{i}^{4}=\bar{\omega}_{i}\left(p_{i} x_{\eta, \varrho q}^{T}+r_{i}\right)
$$

and f nally the output at Layer 5

$$
\Omega^{5}=\left(\overline{\omega_{1}} x_{\eta, \varrho q}^{T}\right) p_{1}+\left(\bar{\omega}_{2} x_{\eta, \varrho q}^{T}\right) p_{2}+\bar{\omega}_{1} r_{1}+\bar{\omega}_{2} r_{2} .
$$

Once the neural fuzzy models have been trained, the signal is easily obtained by taking the original time-domain data $\mathbf{x}$ and introducing them into the two time-domain models $\operatorname{Mod}_{R e}^{T}$ and $\operatorname{Mod}_{I m}^{T}$. Next, after applying a DFT to obtain the frequency-domain signal, it is separated into the four quadrants and introduced into the frequency-domain models $\operatorname{Mod}_{R e, 1 q}, \operatorname{Mod}_{R e, 2 q}, \operatorname{Mod}_{R e, 3 q}, \operatorname{Mod}_{R e, 4 q}$ and $\operatorname{Mod}_{I m, 1 q}$, $\operatorname{Mod}_{I m, 2 q}, \operatorname{Mod}_{I m, 3 q}, \operatorname{Mod}_{I m, 4 q}$ accordingly, to obtain the f nal frequency-domain signal $\mathbf{X}^{T F}$. This way, the obtained signal $\mathrm{f}$ ts the AGP constraints, as it can be seen in Fig. 2 and Fig. 3 for QPSK and 16-QAM, respectively. This TimeFrequency-domain proposal concentrates more the energy than ACE-AGP. The average energy is 1.21 (5.46).

\section{Analysis of Mean Squared Error}

As explained at the beginning of this section, we use a gradient-based learning algorithm to obtain the ANFIS parameters. We consider the following Mean Squared Error (MSE) learning cost function

$$
E=\frac{1}{2} \sum_{m=1}^{q}\left(f_{m}^{d}-f_{m}\right)^{2}
$$

where $q$ is the number of outputs (in our case $q=1$ ), $f_{m}^{d}$ and $f_{m}$ are the desired and the current output values of the network, respectively. Given that the time-domain OFDM signals can be approximated as Gaussian [9], the AGP signal is a truncated Gaussian (to the amplitude threshold $Q$ ) and 
so is the signal obtained by neural fuzzy models. Thus, the Probability Density Function (PDF) of MSE will be

$$
P_{E}(z)=\frac{1}{2} \frac{1}{\sqrt{z}} \frac{\exp \left(-\frac{\left(m_{A N F I S}-\sqrt{z}-m_{A G P}\right)^{2}}{Q\left(\sigma_{A G P}^{2}+\sigma_{A N F I S}^{2}\right)}\right)}{\sqrt{2 \pi Q\left(\sigma_{A G P}^{2}+\sigma_{A N F I S}^{2}\right)}}
$$

where $m_{A G P}, m_{A N F I S}$ are the means of the signals coming from AGP and (time-domain or time-frequency-domain) neural fuzzy systems, respectively, and $\sigma_{A G P}$ and $\sigma_{A N F I S}$ are their variances. Therefore, the CDF (Cumulative Density Function) for MSE can be analytically evaluated by ${ }^{1}$

$$
\begin{aligned}
\operatorname{Prob}(E \leq \varepsilon)= & \operatorname{erf}\left(\frac{m_{A N F I S}-m_{A G P}}{\sqrt{Q\left(\sigma_{A G P}^{2}+\sigma_{A N F I S}^{2}\right)}}\right)- \\
& \operatorname{erf}\left(\frac{m_{A N F I S}-\sqrt{\varepsilon}-m_{A G P}}{\sqrt{Q\left(\sigma_{A G P}^{2}+\sigma_{A N F I S}^{2}\right)}}\right) .
\end{aligned}
$$

The analytical evaluation of the partial derivatives in eq. (10), yields ${ }^{2}$

$$
\begin{gathered}
\frac{\partial E}{\partial p_{i}}=\frac{\partial E}{\partial f} \frac{\partial f}{\partial y_{i}} \frac{\partial y_{i}}{\partial p_{i}}=\left(f-f^{d}\right) x \frac{\mu_{\mathcal{A}_{i}}}{\sum_{l=1}^{n} \mu_{\mathcal{A}_{l}}} \\
\frac{\partial E}{\partial r_{i}}=\frac{\partial E}{\partial f} \frac{\partial f}{\partial y_{i}} \frac{\partial y_{i}}{\partial r_{i}}=\left(f-f^{d}\right) \frac{\mu_{\mathcal{A}_{i}}}{\sum_{l=1}^{n} \mu_{\mathcal{A}_{l}}} \\
\frac{\partial E}{\partial c_{j}}=\frac{\partial E}{\partial f} \frac{\partial f}{\partial \mu_{A_{j}}} \frac{\partial \mu_{A_{j}}}{\partial c_{j}}=\left(f-f^{d}\right) \frac{y_{j}-f}{\sum_{l=1}^{n} \mu_{\mathcal{A}_{l}}} \mu_{\mathcal{A}_{j}} \frac{x-c_{j}}{\sigma_{j}^{2}}
\end{gathered}
$$

and

$$
\frac{\partial E}{\partial \sigma_{j}}=\frac{\partial E}{\partial f} \frac{\partial f}{\partial \mu_{A_{j}}} \frac{\partial \mu_{A_{j}}}{\partial \sigma_{j}}=\left(f-f^{d}\right) \frac{y_{j}-f}{\sum_{l=1}^{n} \mu_{\mathcal{A}_{l}}} \mu_{\mathcal{A}_{j}} \frac{\left(x-c_{j}\right)^{2}}{\sigma_{j}^{3}},
$$

where $f=\frac{\sum_{i=1}^{n} \mu_{\mathcal{A}_{i}} y_{i}}{\sum_{i=1}^{n} \mu_{\mathcal{A}_{i}}}$ and $y_{i}=p_{i} x+r_{i}$.

By using eqs. (20) - (23), eqs. (10) and (11) are solved.

\section{Complexity Analysis}

Once the system has been off-line trained, the operating implementation of the proposed schemes is as simple as introducing the input signal into the generated system model, which performs basically integer multiplications and additions, to obtain the desired signal. The complexity of the Timedomain and the Time-Frequency-domain proposals in terms of number of integer multiplications per OFDM symbol is, respectively, $18 \times N$ and $36 \times N$, while the number of integer additions is, $16 \times N$ and $32 \times N$, respectively. In Table I, a complexity comparison for different schemes and algorithms is summarized. For completeness, the classical Partial Transmit Sequence (PTS) and Selective Mapping (SLM) algorithms and two recent modif ed (and less complex) versions for the PTS [10] and the SLM [11] algorithms have also been included, where $U$ and $M$ are the number of blocks and

\footnotetext{
${ }^{1} \operatorname{erf}(x)=\frac{2}{\sqrt{\pi}} \int_{0}^{x} e^{-t^{2}} d t$.

${ }^{2}$ Since $q=1$, we have removed the sub-index $m$ in the following for clarity purposes.
}

sequences, respectively. Several conclusions can be extracted from the table. The frst one is that our proposals are much less complex than the other schemes, especially because they do not need several (I)DFT operations (the Time-Frequencydomain scheme only needs two). Besides, since our proposals use integer operations, the operations are even simpler ${ }^{3}$ than the other schemes (only complex operations are needed for the Time-Frequency-domain scheme when applying the DFT). In a realistic implementation, the operations can be parallelized and it is possible to perform in parallel the real and imaginary part for each quadrant. The difference in complexity is especially relevant when comparing against ACE-SGP.

\section{RESUlTS}

Monte Carlo simulations have been carried out with 50,000 randomly generated QPSK-modulated or 16-QAM OFDM symbols for $N=256$ and 1024 sub-carriers. The training parts of the experiments in Time-domain and Time-Frequencydomain have been carried out with $70 \%$ data for training and $30 \%$ data for testing. For the ACE-AGP, the maximum number of iterations was $\mathrm{f} x$ ed to be 2, 000 (i.e., a large number of iterations to guarantee its best performance), whereas for the PTS and SLM, the typical values for number of phases and blocks of $2(1,-1)$ (with $U=8$ ) and 16 , respectively.

In Fig. 4, where the BER for the proposed neural fuzzy systems, the original signal and the ACE-AGP were presented, it can be seen that the Time-Frequency-domain ANFIS outperforms the others, and it is close to the original system when only AWGN is present. The reason for this behavior is similar to what was explained for the time-domain scheme: the proposed Time-Frequency-domain ANFIS model concentrates the energy even more than ACE-AGP and moreover, most of the constellation points are moved from their original position, and thus, only a few of symbols will experience an effective lower SNR. Also in this Fig. 4, the performance when the signal is amplifed with a TWTA $^{4}$ is plotted. As it can be seen, the proposed Time-Frequency-domain ANFIS, again, outperforms the other schemes.

In Figs. 5 - 6, several aspects can be observed. The f rst one is that the loss in performance of the proposed TimeFrequency-domain ANFIS scheme with respect to the Timedomain ANFIS system is below $0.3 \mathrm{~dB}$ in terms of CM. The second is that both proposed neural fuzzy schemes outperform the PTS and SLM algorithms. The third one is that the performance loss, in terms of CM, is negligible for $N=256$ and less than $1 \mathrm{~dB}$ for $N=1024$ with QPSK, and it even outperforms ACE-AGP when applied to 16-QAM

\footnotetext{
${ }^{3}$ Typically, integer additions are half complex than complex additions whereas integer multiplications are four times simpler than complex multiplications.

${ }^{4}$ According to Saleh's model [12], the AM/AM conversion can be expressed as

$$
F(|x(t)|)=\frac{\alpha_{||}|x(t)|}{\left(1+\beta_{||}|x(t)|^{2}\right)}
$$

where $\alpha_{\|}$is the small signal gain, $A_{\text {sat }}=\frac{1}{\sqrt{\beta_{||}}}$is the input saturation voltage of TWTA and $A_{\max }=\frac{\alpha_{\|}}{2 \sqrt{\beta_{\|}}}=\frac{\alpha_{\|} A_{\text {sat }}}{2}$ stands for the maximum output amplitude. For the results, only AM-AM conversion has been taken into account, and $\alpha_{\|}=2, \beta_{\|}=1$. A value of Input Back Off (IBO) $=10$ $\mathrm{dB}$ was used.
} 
TABLE I

COMPLEXITY SUMMARY AND COMPARISON FOR DIFFERENT METHODS. NOTE THAT TYPICALLY, INTEGER ADDITIONS ARE HALF COMPLEX THAN COMPLEX ADDITIONS WHEREAS INTEGER MULTIPLICATIONS ARE FOUR TIMES SIMPLER THAN COMPLEX MULTIPLICATIONS.

\begin{tabular}{|c|c|c|c|c|c|c|c|}
\hline & $\begin{array}{l}\text { Original } \\
\text { SLM }(U= \\
16)\end{array}$ & $\begin{array}{l}\text { Modified } \\
\text { SLM [11] } \\
(\mathbf{U}=4)\end{array}$ & $\begin{array}{l}\text { Original } \\
\text { PTS }(U=8, \\
M=64)\end{array}$ & $\begin{array}{l}\text { Modified PTS } \\
{[10](U=8, M} \\
=16)\end{array}$ & $\begin{array}{l}\text { ACE-SGP } \\
\left(N_{\text {iter }}=50\right)\end{array}$ & $\begin{array}{l}\text { Time } \\
\text { Domain }\end{array}$ & $\begin{array}{l}\text { Time-Frequency } \\
\text { Domain }\end{array}$ \\
\hline (I)DFT & 16 & 4 & 64 & 16 & 100 & 0 & 2 \\
\hline \# Complex Mult & 20480 & $\overline{8192}$ & 10020 & $\overline{5376}$ & 51200 & - & 2048 \\
\hline \# Complex Adds & 32768 & 11264 & 200704 & 54272 & 102400 & - & 4096 \\
\hline \# Integer Mult. & - & - & - & - & - & 4608 & 9216 \\
\hline \# Integer Adds & - & - & - & - & - & 4096 & 8192 \\
\hline \multicolumn{8}{|c|}{$\bar{N}=1024$} \\
\hline \#\# Complex Mult & 98304 & 36864 & 40960 & 24192 & 204800 & - & 10240 \\
\hline \# Complex Adds & 163840 & 77824 & 1003520 & 249652 & 409600 & - & 20480 \\
\hline \# Integer Mult. & - & - & - & - & - & 18432 & 36864 \\
\hline \# Integer Adds & - & - & - & - & - & 16384 & 32768 \\
\hline \# Check operations & 16 & 16 & 64 & 16 & 50 & - & - \\
\hline
\end{tabular}

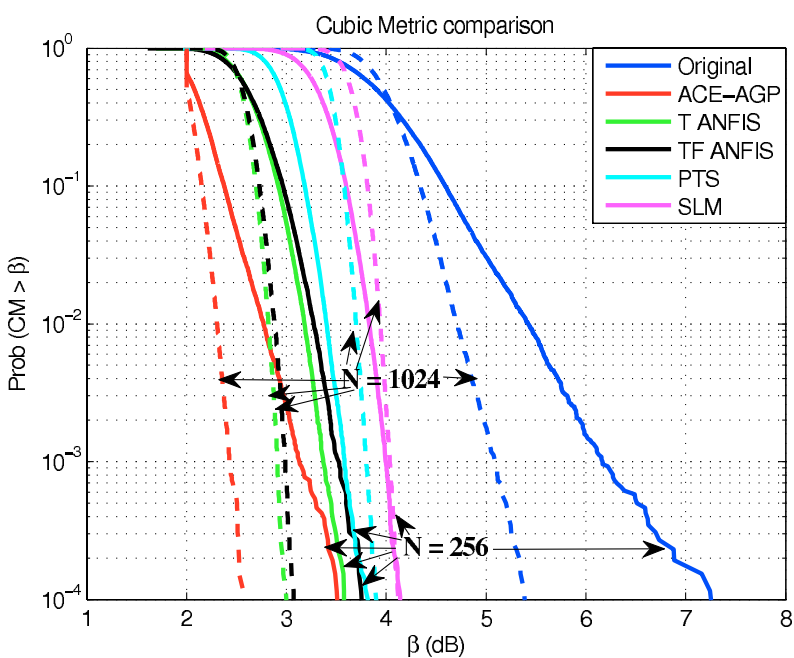

Fig. 5. Comparison of $\mathrm{CM}$ reduction results using QPSK with $\mathrm{N}=256$ (solid) and 1024 (dashed)

modulation (the convergence of ACE-AGP for higher order modulations is slower). We would like to emphasize that all these advantages are achieved without the complexity and large required number of iterations of the conventional ACEAGP or other methods.

Besides, it is shown in [13] that the degradation in the reduction of envelope fuctuations (not in BER performance) of including pilot sub-carriers is less than $0.3 \mathrm{~dB}$ for realistic pilot density.

Finally, in Fig. 7, the analytical CDF in eq. (19) for the MSE, evaluated with values of $m_{A G P}=2.72 \cdot 10^{-5}$, $m_{A N F I S}=2.98 \cdot 10^{-5}, \sigma_{A G P}=1.3 \cdot 10^{-3}, \sigma_{A N F I S}=$ $1.2 \cdot 10^{-3}$ and $Q=7.3 \cdot 10^{-2}$, is shown and compared to the MSE obtained by simulations. It can be observed that the MSE is very small and the differences with respect to the simulations are negligible.

\section{CONCLUSION}

In this paper, two novel methods for the reduction of the envelope fuctuations in multi-carrier signals have been proposed. It has been shown that the proposed Time-domain

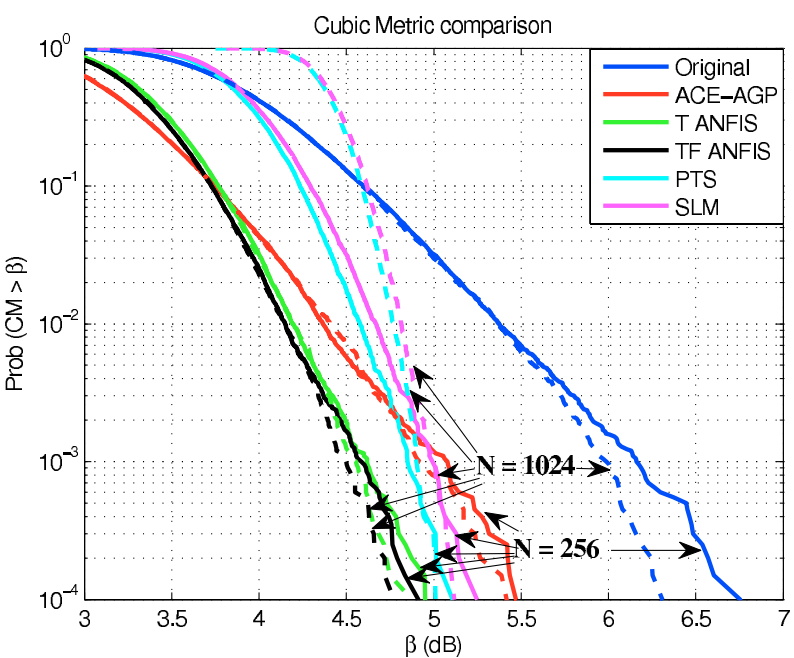

Fig. 6. Comparison of $\mathrm{CM}$ reduction results using 16-QAM with $\mathrm{N}=256$ (solid) and 1024 (dashed)

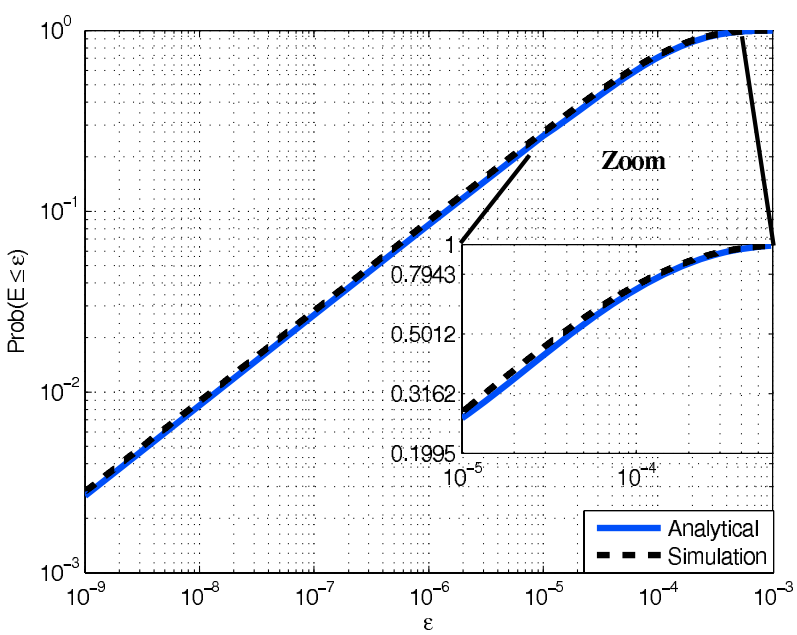

Fig. 7. $\mathrm{CDF}$ for the MSE for values $m_{A G P}=2.72 \cdot 10^{-5}, m_{A N F I S}=$ $2.98 \cdot 10^{-5}, \sigma_{A G P}=1.3 \cdot 10^{-3}, \sigma_{A N F I S}=1.2 \cdot 10^{-3}$ and $Q=7.3 \cdot 10^{-2}$

neural Fuzzy system, although simpler than Time-Frequencydomain, offers a good performance in terms of reduction of envelope fuctuations but not in terms of BER. On the other hand, 
the proposed Time-Frequency-domain neural fuzzy model offers similar reductions as Time-domain scheme and better performance in terms of BER, at the expense of a small increase in system complexity. The analytically obtained MSE has been shown to be very small and match accurately the simulations. It has been shown that both proposals outperform other already proposed schemes with a reduced complexity.

\section{REFERENCES}

[1] 3rd Generation Partnership Project (3GPP), "UTRA-UTRAN long term evolution (LTE) and 3GPP system architecture evolution (SAE)." [Online]. Available: http://www.3gpp.org/Highlights/LTE/LTE.htm, Accessed on 2 July 2009.

[2] S. H. Han and J. H. Lee, "An overview of peak-to-average power ratio reduction techniques for multicarrier transmission," IEEE Wireless Commun., vol. 12, no. 2, pp. 56-65, Apr. 2005.

[3] B. S. Krongold and D. L. Jones, "PAR reduction in OFDM via active constellation extention," IEEE Trans. Broadcasting, vol. 49, no. 3, pp. 258-268, Sep. 2003.

[4] J. S. R. Jang, "Adaptive-network-based fuzzy inference system," IEEE Trans. Systems, Man Cybernetics, vol. 23, no. 3, pp. 665-685, 1993.

[5] P. J. Pacini and B. Kosko, "Adaptive fuzzy frequency hopper," IEEE Trans. Commun., vol. 43, no. 6, pp. 2111-2117, June 1995.
[6] J. Wang and J. Chen, "Performance of wideband CDMA systems with complex spreading and imperfect channel estimation," IEEE J. Sel. Areas Commun. (JSAC), vol. 19, no. 1, pp. 152-163, Jan. 2001.

[7] TDoc R1-060023, "Cubic Metric in 3GPP-LTE," 3GPP TSG RAN WG1, Tech. Rep., Jan. 2006.

[8] L. E. Aik, S. Yogan, and O. Jayakumar, "A study of neuro-fuzzy system in approximation-based problems," MATEMATIKA, vol. 23, no. 2, pp. 113-130, 2008.

[9] H. Ochiai and H. Imai, "On the distribution of the peak-to-average power ratio in OFDM signals," IEEE Trans. Commun., vol. 49, no. 2, pp. 282289, Feb. 2001

[10] H.-Y. Tseng, Y.-H. Chung, S.-M. Phoong, and Y.-P. Lin, "A reduced complexity PTS scheme for peak-to-average power ratio reduction in OFDM systems," in Proc. EUSIPCO, 2008.

[11] S.-J. Heo, H.-S. Noh, J.-S. No, and D.-J. Shin, "A modif ed SLM scheme with low complexity for PAPR reduction of OFDM systems," IEEE Trans. Broadcasting, vol. 53, no. 4, pp. 804-808, Dec. 2007.

[12] A. A. M. Saleh, "Frequency-independent and frequency-dependent nonlinear models of TWT amplif ers," IEEE Trans. Commun., vol. 29, no. 11, pp. 1715-1720, Nov. 1981.

[13] Y. Jabrane, V. P. G. Jiménez, A. G. Armada, B. E. Said, and A. A. Ouahman, "Evaluation of the effects of pilots on the envelope fuctuations reduction based on neural fuzzy systems," in Proc. IEEE International Workshop Signal Process. Advances Wireless Commun. (SPAWC), vol. 1, Marrakech, Morocco, June 2010. 